\title{
Reception of Morse code through motional, vibrotactile, and auditory stimulation
}

\author{
HONG Z. TAN, NATHANIEL I. DURLACH, WILLIAM M. RABINOWITZ, \\ CHARLOTTE M. REED, and JONATHAN R. SANTOS \\ Massachusetts Institute of Technology, Cambridge, Massachusetts
}

\begin{abstract}
The potential for communication through the kinesthetic aspect of the tactual sense was examined in a series of experiments employing Morse code signals. Experienced and inexperienced Morse code operators were trained to identify Morse code signals that were delivered as sequences of motional stimulation through up-down displacements (roughly $10 \mathrm{~mm}$ ) of the fingertip. Performance on this task was compared with that obtained for both vibrotactile and acoustic presentation of Morse code using a 200-Hz tone delivered either to the fingertip through a minishaker or diotically to the two ears under headphones. For all three modalities, the ability to receive Morse code was examined as a function of presentation rate for tasks including identification of single letters, random three-letter sequences, common words, and sentences. Equivalent word-rate measures (i.e., product of percent correct scores and stimulus presentation rate) were nearly twice as high for auditory presentation as for vibrotactile stimulation, which in turn was about 1.3 times that for motional stimulation. The experienced subjects outperformed the inexperienced subjects by amounts that increased with task complexity. For example, the former were able to receive sentences at 18 words $/ \mathrm{min}$ with motional stimulation, whereas the latter, following $75 \mathrm{~h}$ of training, were unable to perform this task. The present results and those of other research with tactual communication systems are compared, particularly regarding estimates of information-transfer rates.
\end{abstract}

In this paper, we focus on the ability to receive information through motional stimulation (i.e., the kinesthetic sense). Our long-term goals are (1) to study the kinesthetic sense as a communication channel, (2) to compare performance through the kinesthetic sense with that through other senses, and (3) to compare the ability to receive motional stimulation with the ability to produce the same movement patterns.

Most studies of tactual communication have focused on the cutaneous/tactile sensory system (see Geldard, 1973; Kaczmarek, Webster, Bach-y-Rita, \& Tompkins, 1991). In contrast, research on the kinesthetic sensory system is limited (see Clark \& Horch, 1986, for a review), and few devices have been designed to stimulate the kinesthetic sense. However, two systems are noteworthy because they were designed to stimulate the kinesthetic sense for purposes of tactual communication.

In a creative study, Bliss (1961) developed an air-driven finger stimulator that was employed as a "reverse typewriter." The stimulator consisted of eight finger rests arranged in two groups on which the user could place the

This research was supported by Research Grant 2 R01 DC 00126-16 from the National Institute on Deafness and Other Communication Disorders, National Institutes of Health. The authors are greatly indebted to Phil Temples (call sign K9HI) and Joe Parskey (call sign NJIP) for their expertise and dedication in working as the experienced subjects. Correspondence should be addressed to H. Z. Tan, MIT, The Media Laboratory, Room E1 5-384E, 20 Ames St., Cambridge, MA 02139 (e-mail: hongtan@media.mit.edu). fingers of both hands in a manner similar to that of the "home" position of a typewriter. Each stimulator was capable of simulating motions corresponding to the active movements of a typist's fingers in reaching the upper and lower rows on a keyboard. In one set of experiments, 42 random triplets composed of the letters $e, t, n, a, o$, and $i$ were presented to 8 subjects. The average information transfer was 1.75 bits/letter out of a maximum possible $2.58 \mathrm{bits} / \mathrm{letter}$. In another experiment, 30 symbols (the alphabet, comma, period, space, and uppercase) were presented in random order with equal probability to 1 subject (with less than $15 \mathrm{~h}$ of practice). Six sequences of 130 symbols each were delivered at a rate of $0.5-1.5$ letters/ sec. The subject responded verbally by naming the symbols as they were received. The information rate, computed as the product of percent correct, presentation rate (letter/sec), and information per symbol (4.91 bits/letter), reached a maximum of $4.5 \mathrm{bits} / \mathrm{sec}$ at a presentation rate of 1.32 letters/sec.

Eberhardt, Coulter, Bernstein, Barac-Cikoja, and Jordan (1994) developed a 2-degree-of-freedom (up-down and front-back) finger stimulator ("OMAR") that could provide motional and vibrational stimulation to a finger through a single actuator. Initial experiments demonstrated that subjects were able to judge onset asynchronies of vibration and movement with the system.

The main purpose of the present study was to investigate the feasibility of utilizing the kinesthetic sense for communication. Specifically, we wanted to study the ability to receive information through up-down finger mo- 
tions and to compare directly performance through the motional (i.e., kinesthetic) condition with that obtained through the tactile/cutaneous and auditory modalities. To assess the communication rate, a code was needed that could represent all three types of stimulation as meaningful messages. The International Morse Code was chosen because it is a well-established code with learning patterns that have been well studied. Bryan and Harter (1899) followed students of telegraphy for over half a year and tested their ability to send and receive Morse code weekly. They found that while the students' ability to send the code improved monotonically, their ability to receive the code reached several plateaus and eventually exceeded that of sending. The plateaus in the reception curves were interpreted as evidence that a student of telegraphy first learned to receive individual letters, then developed the skills to receive common words as the basic units, and eventually learned to receive short phrases after many years of practice.

In our study, highly skilled Morse code operators were included as subjects in an attempt to exploit their previous experience in chunking coded messages. We hoped that their previous experience in chunking auditory Morse code would transfer to the tactual domain and thereby circumvent the long training periods that might otherwise be required to process long stimulating streams (e.g., as in sentences with a completely novel code). Inexperienced subjects were also trained and tested for comparison. The fact that Morse code is used both to send and to receive information also enabled us to investigate the relationship between the ability to receive motional stimulation and the ability to produce such motions. Finally, Morse code can be adapted to many sensory modalities. Although Morse code is traditionally received through the auditory channel, hearing-impaired ham operators have put their hands on speakers to receive Morse code through the tactual channel. We compared subjects' ability to receive the Morse code through motional, vibrotactile, and auditory stimulation using common tasks.

\section{METHOD}

Morse code is a temporal sequence of patterns in which each letter of the alphabet has its own unique pattern. Patterns consist of elements (dot $=$ one unit $=U$; dash $=$ three units $=3 U$ ) and pauses. Morse code reception was studied for motional, vibrotactile, and auditory stimulation as a function of presentation rate $(R)$ in words per minute (wpm), which is related to the duration of $U$ (in milliseconds) by $R=1,200 / U$. A more complete description of Morse code is provided in the Appendix.

\section{Subjects}

Two experienced Morse code operators from the Boston Amateur Radio Club (Subjects El and E2) and 2 inexperienced MIT students (Subjects N1 and N2) participated in the experiments.' $\mathrm{E} 1$ and E2 were both males, 38 and 40 years of age, and were licensed as extraclass ham radio operators. $\mathrm{N} 1$ was a 28 -year-old female, and $\mathrm{N} 2$ was an 18-year-old male. Three of the subjects (E2, N1, and N2) were right-handed, and $I(E l)$ was left-handed. Except for N1, who was also the experimenter, the subjects were paid on an hourly basis.

\section{Tasks}

The reception of Morse code through motional, vibrotactile, and auditory stimulation was studied using four tasks in the following order: single-letter identification, three-letter random-sequence identification, common-word identification, and sentence reception. The first two tasks were designed to train the subjects to achieve certain performance goals with letter-based materials. The last two tasks were designed to test the subjects with word-and sentencebased English materials. Table 1 lists the testing conditions in chronological order. For Subjects E1 and E2, all tasks were completed first under motional stimulation, followed by the vibrotactile condition, and finally the auditory condition. For Subjects N1 and N2, the motional condition for all tasks was completed first; vibrotactile and auditory conditions were then alternated within each of the tasks. All 4 subjects participated in each experiment except that (1) the experienced subjects were not trained auditorily with the single-letter and three-letter sequences (because these tasks were trivial given these subjects' previous experience with auditory reception of Morse code) and (2) only the experienced subjects were tested with sentences (because the inexperienced subjects were unable to perform this task).

Training of single-letter identification. On each trial, the subject was presented (through motional, vibrotactile, or auditory stimulation, as described below) with the Morse code for 1 of the 26 letters of the English alphabet. The subject was instructed to respond with 1 of the 26 letters on a computer keyboard, and then trial-bytrial correct-answer feedback was provided by the displaying of the correct response on a computer screen. Each run consisted of 130 presentations of single letters in random order with each of the 26 letters presented exactly five times. The duration of each run varied from 5 to $20 \mathrm{~min}$, depending on the response time of the subject. Each subject started from the lowest rate of stimulus presentation, and each was allowed to proceed to the next higher rate only after one of the following criteria was achieved: (1) one run with a perfect score of $100 \%,(2)$ at least three runs with scores over $95 \%$ (not necessarily consecutively), or (3) roughly 10 or more consecutive runs with similar scores (i.e., a clear plateau). Four rates were used: $12,16,20$, and $24 \mathrm{wpm}$, except for motional stimulation where the rate of $20 \mathrm{wpm}$ was not used.

Training of three-letter identification. On each trial, the subject was presented (through motional, vibrotactile, or auditory stimulation) with the Morse code of a three-letter nonsense word (with each letter chosen randomly with equal a priori probabilities from the 26 letters). The subject was instructed to respond with a three-letter sequence and then shown the correct response. The letters were separated by a pause of duration $3 U$ (see Figure $\mathrm{A} 2$ in the Appendix). The subject could either "copy on the fly" (i.e., the subject entered the response to the first letter while the second letter was being presented)

Table 1

Experimental Conditions and Ordering

\begin{tabular}{lll}
\multicolumn{1}{c}{ Mode } & \multicolumn{1}{c}{ Task } & \multicolumn{1}{c}{ Subjects } \\
\hline$M$ & one-letter & E1, E2, N1, N2 \\
$M$ & three-letter & E1, E2, N1, N2 \\
$M$ & words & E1, E2, N1, N2 \\
$M$ & sentences & E1, E2 \\
V & one-letter & E1, E2 \\
V\&A interleaved & one-letter & N1, N2 \\
V & three-letter & E1, E2 \\
V\&A interleaved & three-letter & N1, N2 \\
V & words & E1, E2 \\
V\&A interleaved & words & N1, N2 \\
V & sentences & E1, E2 \\
A & sentences & E1, E2 \\
A & words & E1, E2 \\
\hline
\end{tabular}

Note- $M$, motional; $V$, vibrotactile; $A$, auditory. 
or "copy behind" (i.e., the subject waited until all three letters were presented before entering the response). All subjects chose the copybehind method of responding for this training task. Each run consisted of 52 presentations of three-letter sequences in random order, such that each letter of the alphabet was presented exactly six times. A response was considered correct only if all three letters were identified correctly in the correct order. Each subject started from the lowest rate of stimulus presentation, and each was allowed to proceed to the next higher rate only after one of the following criteria was achieved: (1) one run with a perfect score of $100 \%,(2)$ three runs with scores over $90 \%$ (not necessarily consecutively), or (3) roughly 10 or more consecutive runs with similar scores (i.e., a clear plateau). Four rates were used: 12, 16, 20, and 24 wpm.

Test of common-word identification. The material consisted of 600 words obtained from the corpus of The American Heritage Word Frequency Book (Carroll, Davies, \& Richman, 1971). The selection of words was based on rate of occurrence and minimum length. All the stimuli occupy ranks between 1,000 and 5,300 per million and contain at least seven letters. Two randomizations of the 600 words into twelve 50-item word lists were constructed and employed in the testing, such that all lists from the first randomization were presented prior to lists from the second randomization. The subjects were told before the experiment that the test material consisted of common English words. On each trial, the subject was presented (through motional, vibrotactile, or auditory stimulation) with the Morse code of one word from a chosen list, instructed to respond by typing out a response word (either by "copying on the fly" or by "copying behind"), and then shown whether the response was "right" or "wrong." 2 The letters within a word were separated by a pause of duration $3 U$ (see Figure A2 in the Appendix). Each run consisted of one list (i.e., 50 words) presented with the same stimulation mode and presentation rate. Different rates were selected for experienced and inexperienced subjects with each of the three types of stimulation in order to obtain a wide range of percent correct scores as a function of stimulus presentation rate. Each subject performed three runs per stimulus presentation rate and proceeded from the lowest to the highest rate. (This was the only task where all subjects were tested with all three modes of stimulation.)

Test of sentence reception. The test material consisted of CUNY sentence lists commonly used for speech and hearing research (Boothroyd, Hanin, \& Hnath, 1985). Each of the 60 lists contains 12 sentences arranged by topic (e.g., food, animals, weather, etc.). Each sentence in a list consists of 3-14 common English words, and each list contains exactly 102 words. The same list was never used twice with the same subject. The difficulty levels of these sentences were estimated to be equivalent to fifth or sixth grade reading levels. Prior to the experiment, the subjects were told that the test material consisted of conversational sentences but were not informed of the topics. On each trial, the subject was presented (through motional, vibrotactile, or auditory stimulation) with the Morse code of one sentence from a chosen list, instructed to repeat the sentence verbally, and given only informal feedback (e.g., the experimenter revealed specific words in the sentence if the subject asked). Letters within a word were separated by a pause of duration $3 U$, and words within a sentence were separated by a pause of duration $7 U$ (see Figure $\mathrm{A} 2$ in the Appendix). The subject could either respond "on the fly" or after the entire sentence had been presented. Each run consisted of one list (i.e., 12 sentences) presented with the same stimulation mode and presentation rate. At the end of a run, the experimenter counted the number of words that the subject was able to repeat regardless of the ordering and ignored extra words in the response. The overall word score was computed as the number of correctly repeated words divided by 102 , the total number of words in each CUNY sentence list. Different rates were selected for the three types of stimulation in order to obtain a wide range of percent correct scores as a function of stimulus presentation rate. Each subject was tested with three lists at each rate, and each subject proceeded from the lowest to the highest rate.

During all experiments, the subject was informed of the overall percent correct score at the end of each run. Each experimental session lasted $1-2 \mathrm{~h}$. The subjects were free to take breaks between runs at their own pace. The experienced subjects generally completed two sessions per week. The inexperienced subjects completed three or more sessions per week.

\section{Instrumentation and Procedure}

Motional stimulation. A device designed to move the fingertip up and down was constructed around a permanent magnet servomotor with feedback from a tachometer and an optical encoder (Figure 1). A Plexiglas lever was attached to the motor shaft. The subject rested the index fingertip lightly over a roller that was snugfit into a hole on the lever. The distance from the center of the motor shaft to that of the roller was $40 \mathrm{~mm}$. The roller served to control the point of contact and to accommodate any relative motions between the finger and the lever. The system parameters were adjusted so that the position-step response was monotonic (critically damped) with a rise/fall time (i.e., time between $10 \%$ and $90 \%$ of full displacement) of approximately $20 \mathrm{msec}$. This rise/fall time limited the highest presentation rate that could be delivered by the motor.

The waveforms used to drive the motor were two-level square waves. Figure 2 shows the waveform for the letter $P$ along with its Morse code. Each waveform started with an interletter pause of $3 U$ followed by the appropriate dot-dash pattern for that letter. For the typical arrangement of the stimulator system, a downward motion at the fingertip indicated the onset of a dot or a dash. The actual vertical displacement of the fingertip was adjusted to be about $10 \mathrm{~mm}$. In preliminary experiments, this value was found to be the largest amplitude that felt comfortable at the highest rate tested (i.e., $24 \mathrm{wpm}$ ). With the finger pressing lightly on the roller, the overall position of the roller (and lever) shifted downwards by $1-2 \mathrm{~mm}$, but the relative up-down motion was otherwise unchanged.

The apparatus was always hidden from view. The subjects wore earphones with acoustic noise to mask any auditory cues from the apparatus. Stimuli were presented to the index finger of the dominant hand of each subject. The standard posture was to rest the fingertip lightly on top of the roller and follow the up-down motions of the roller. In general, the subjects were encouraged to use a consistent posture throughout all experiments, although alternative postures were employed by some subjects under some conditions. ${ }^{3}$ The range of presentation rates was 4-24 wpm across the various tasks, corresponding to a range in $U$ of $300-50 \mathrm{msec}$. Before the experiments began, the inexperienced subjects were provided with a brief training period (averaging $3.6 \mathrm{~h}$ ) to associate letters with the movement patterns.

Vibrotactile stimulation. Stimulation was applied through an electrodynamic minishaker (Alpha-M AV-6). A 200-Hz sinusoidal signal gated by the square wave shown in Figure 2 was applied to the minishaker. The rise time of the minishaker in response to a $200-\mathrm{Hz}$ pulse was measured to be less than $4 \mathrm{msec}$. The presence and duration of the vibration indicated the presence and duration of a dot or a dash. The subject placed the index finger of the dominant hand on the top of a flat contactor ( $9 \mathrm{~mm}$ in diameter) that was fit to the minishaker. The range of presentation rates was $8-40 \mathrm{wpm}$ across the various tasks, corresponding to a range in $U$ of 150 $30 \mathrm{msec}$. The amplitude of the $200-\mathrm{Hz}$ vibration was held constant at roughly $56-\mathrm{dB}-\mu \mathrm{m}$ peak across presentation rates, corresponding to a range of sensation levels of roughly 45-50 dB SL across the signal-duration range of $30-150 \mathrm{msec}$ employed here (see Gescheider, Hoffman, Harrison, Travis, \& Bolanowski, 1994, for a discussion of the effects of signal duration on vibrotactile threshold; see Verrillo \& Smith, 1976, for a discussion of the effects of duration on sensation magnitude). 


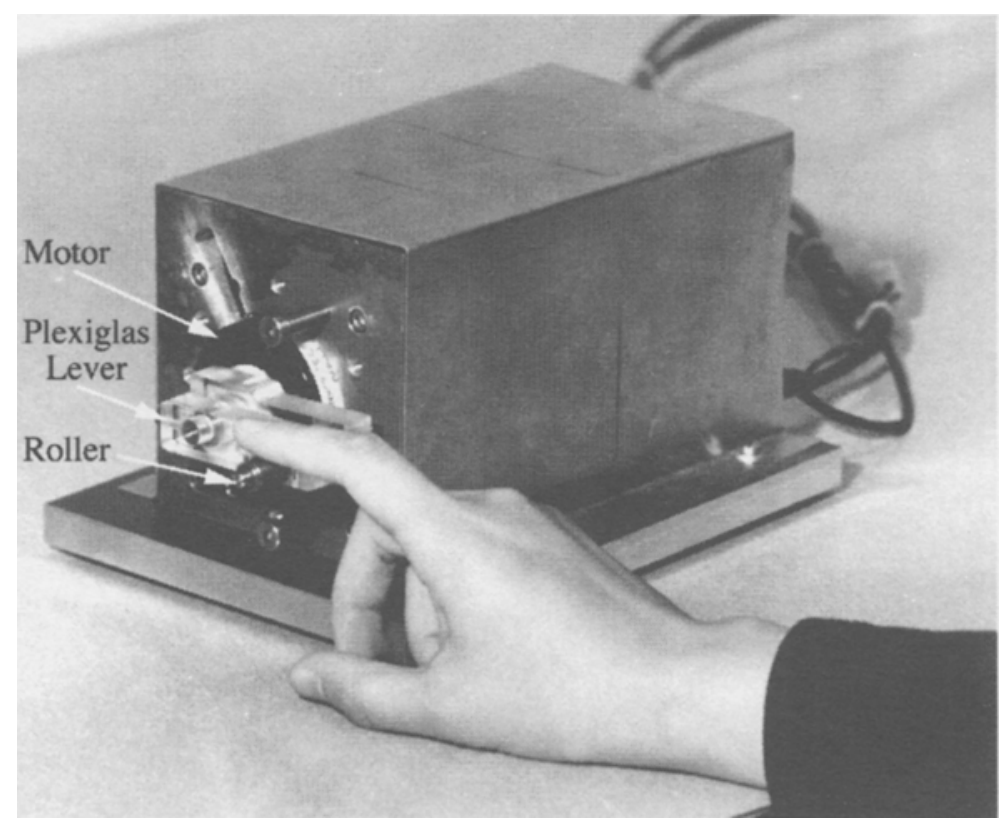

Figure 1. The experimental apparatus. The finger is rested on a roller placed $40 \mathrm{~mm}$ from the center of the rotor. The two shoulder screws above and below the Plexiglas bars serve as the mechanical stops.

During the experiments, the minishaker was placed inside a wooden box lined with sound-absorbing foam to shield it visually from the subject and to attenuate the sound caused by the vibration. The subjects wore earphones with acoustic noise to mask any residual auditory cues from the minishaker.

Auditory stimulation. Morse code sequences were presented diotically via headphones using the same $200-\mathrm{Hz}$ signals that were applied to the minishaker. The presence and duration of an auditory tone indicated the presence and duration of a dot or a dash. For stimulus presentation rates above $56 \mathrm{wpm}$, a 5 -msec smoothing window was applied to the rising and falling portion of the signals to reduce "clicks." The subject could adjust the overall gain so that the earphone signal "felt comfortably loud." The range of presentation rates was $12-73.85 \mathrm{wpm}$ across the various tasks, corresponding to a range in $U$ of $100-16.25 \mathrm{msec}$.

\section{Data Analysis}

For the training tasks, learning curves were constructed by plotting the percent correct scores as a function of run number for each subject, task, type of stimulation, and presentation rate. Decisions on when to terminate the training were made on the basis of the performance criteria described in the Tasks subsection within the Method section. The number of runs required to meet criterion performance for each subject and presentation rate is presented in Table 2. The learning-curve data were then reduced by averaging percent correct scores over the final three runs at each presentation rate. For the test tasks, the percent correct scores from the three runs conducted at each experimental condition were averaged. Statistical analyses were applied to a subset of the data where appropriate.

\section{RESULTS}

\section{Single-Letter Identification}

Learning curves for motional stimulation are shown in Figure 3 for each of the 4 subjects at each of the three pre- sentation rates. Because training progressed from presentation rates of 12 to 16 to $24 \mathrm{wpm}$, the learning rates observed at 16 and 24 wpm may have been influenced by the previous training at the slower rates. Apparently, the change in movement direction for Subject El and the increase in movement amplitude for Subject E2 had little effect on the overall characteristics of the learning curves at $24 \mathrm{wpm}$ for the 2 experienced subjects (see note 3 ). Learning curves for Subjects E1, E2, and N1 were quite similar at each of the three presentation rates both in terms of the number of trials required to meet criterion performance and in the final levels of performance. Although the performance of Subject N2 was similar to that of the other subjects at 12 and $16 \mathrm{wpm}$, his learning curve at the highest rate of $24 \mathrm{wpm}$ indicates that he could not achieve $95 \%$ correct performance even after about 60

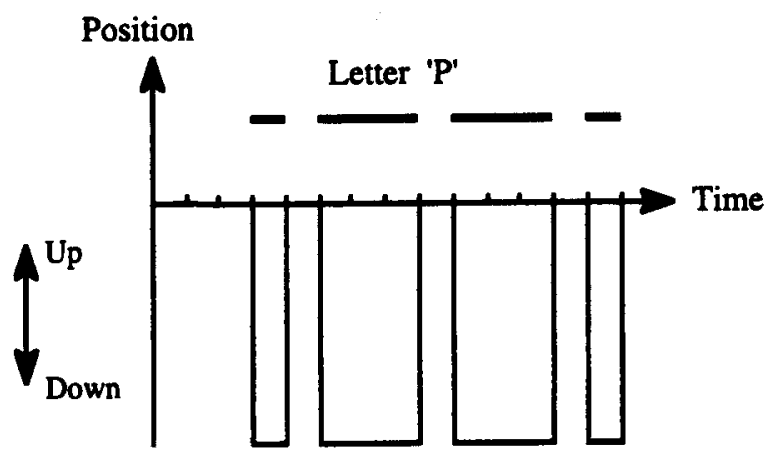

Figure 2. Waveform used to deliver the letter $P$ for motional stimulation. 

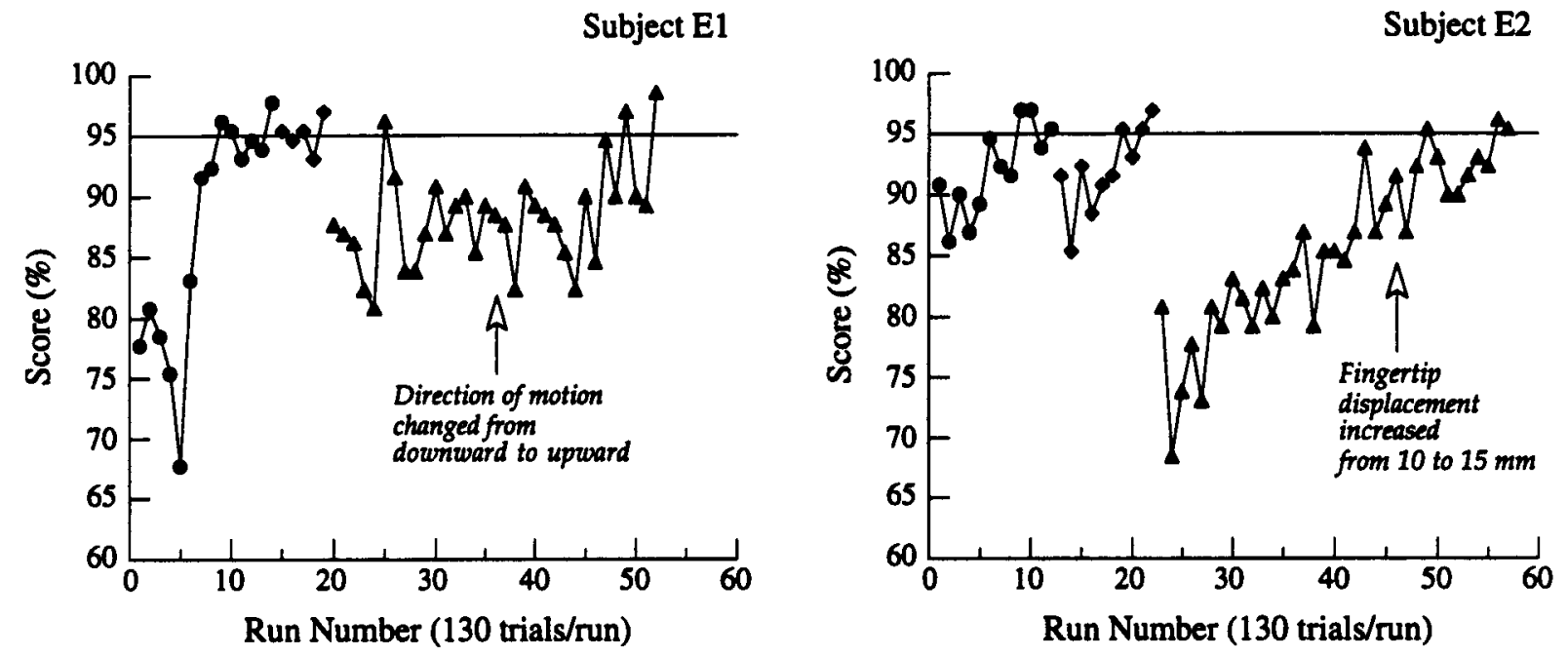

\section{Subject N1}
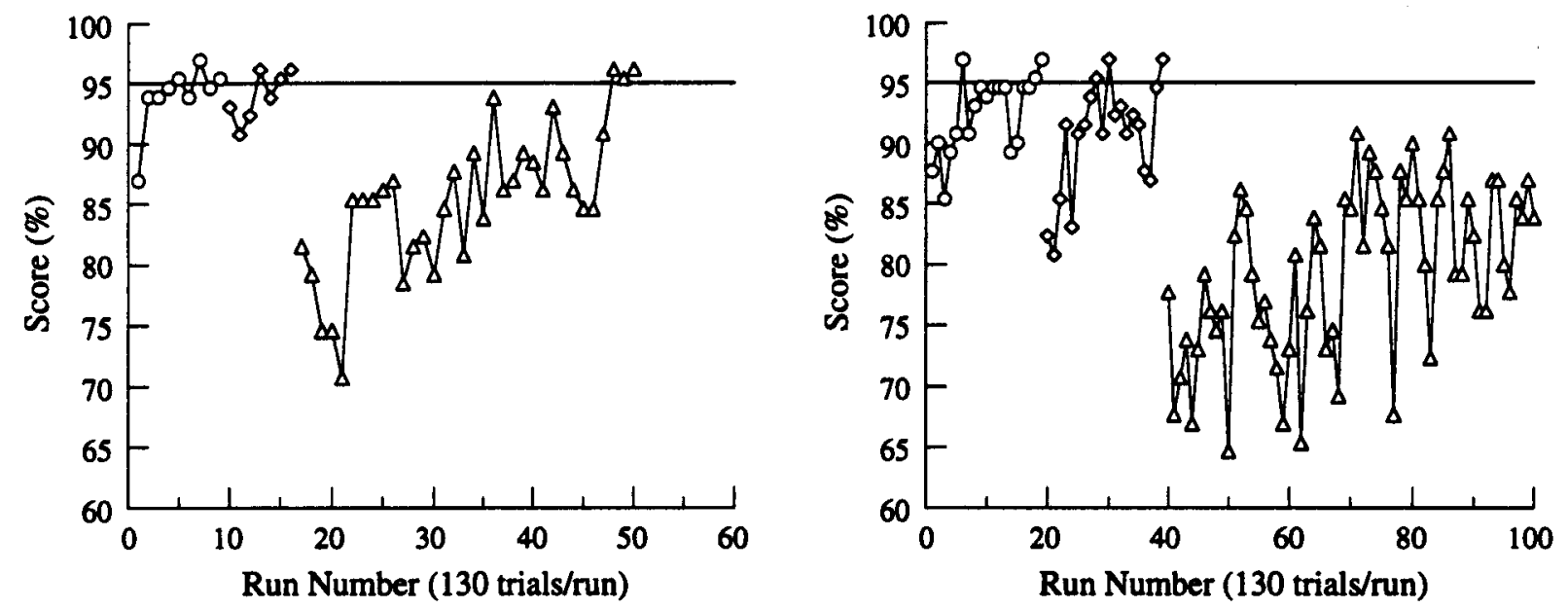

Figure 3. Learning curves for motional stimulation from single-letter identification training for all subjects (E1 and E2, filled symbols, above; N1 and N2, open symbols, below) at 12 wpm (circles), $16 \mathrm{wpm}$ (diamonds), and 24 wpm (triangles). Horizontal lines indicate the performance level of $95 \%$.

runs at that rate (asymptoting instead at roughly $85 \%-$ $90 \%$ performance). It should be pointed out that Subject N1 was also the experimenter and thus was highly familiar with the signals and the task before formal data collection began. Thus, for this particular task, Subject N2 was the only subject who had no prior experience with either Morse code or motional stimulation.

The learning curves for vibrotactile and auditory stimulation were similar to those shown for motional stimulation. The number of runs required to meet criterion performance for each subject, presentation rate, and stimulation mode is presented in Table 2 . The percent correct scores averaged over the last three runs for motional, vibrotactile, and auditory stimulation are shown in Figure 4. For motional stimulation, the performance levels of Subjects E1 and E2 were similar: Both performed above $95 \%$ correct at 12 and $16 \mathrm{wpm}$ and slightly below $95 \%$ correct at $24 \mathrm{wpm}$. Subject N2 reached plateaus of $96 \%$, $93 \%$, and $85 \%$ correct at 12,16 , and $24 \mathrm{wpm}$, respectively. Only Subject N1 performed above $95 \%$ correct at all three rates of motional stimulation, thereby demonstrating that (for this relatively simple task) performance was mainly dependent on the experience with the up-down motional signals rather than prior knowledge of Morse code. With vibrotactile stimulation, 3 of the 4 subjects (E1, E2, and N1) were able to perform above $95 \%$ correct at all four rates tested, but Subject N2 was not able to do so at rates above $16 \mathrm{wpm}$. Specifically, Subject N2 reached plateaus of $92 \%$ and $73 \%$ at 20 and $24 \mathrm{wpm}$, respectively. With auditory stimulation, both inexperienced subjects were able to reach near-perfect scores at all rates tested. ${ }^{4}$ Criterion performance for Subjects N1 and N2 was achieved much more rapidly for auditory stimulation than for vibrotactile stimulation (see Table 2). Over- 

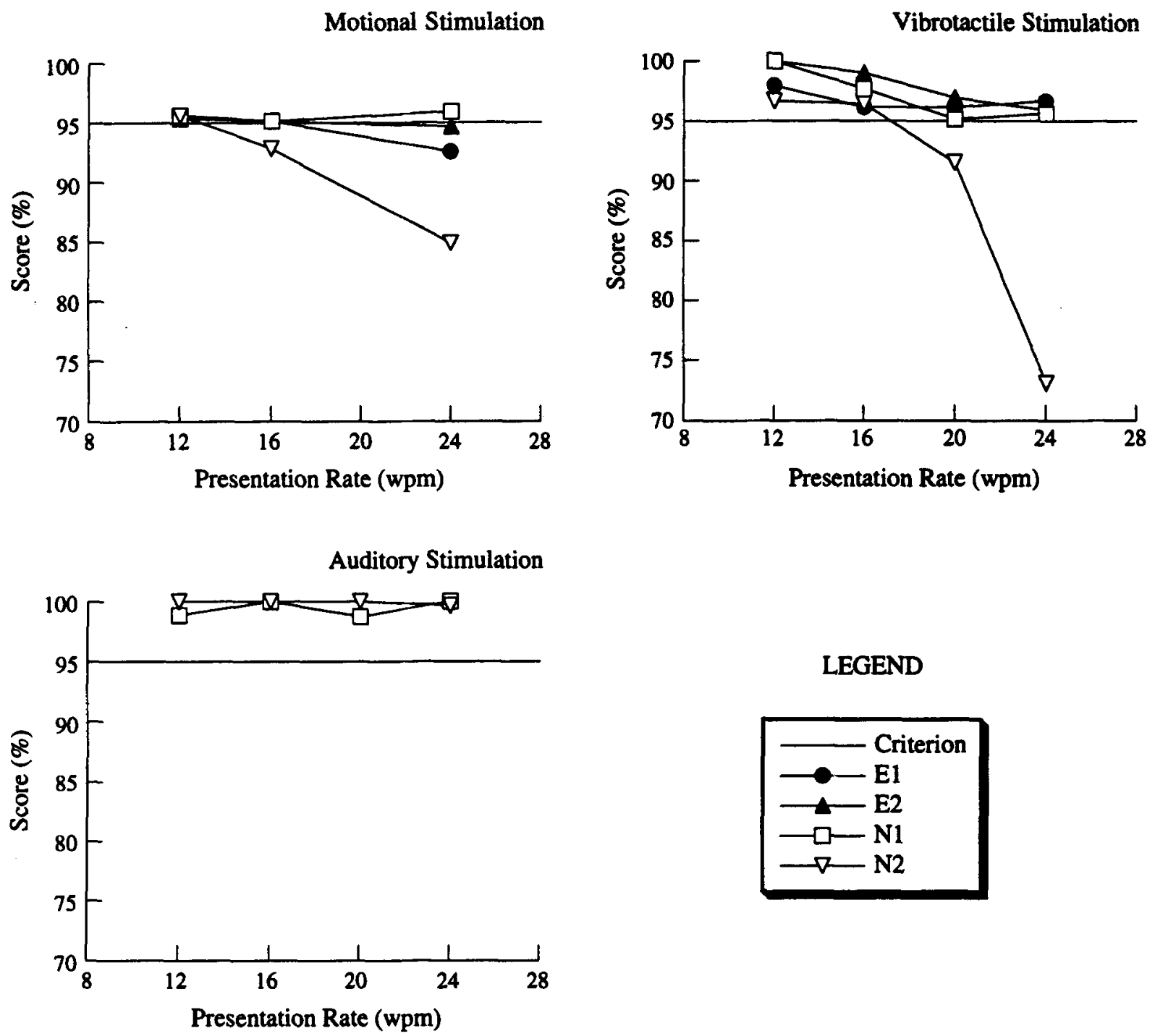

\section{LEGEND}

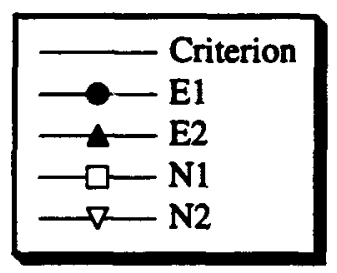

Figure 4. Percent correct scores averaged over the final three runs from single-letter identification training as a function of presentation rate. Individual subject results are shown in separate panels for motional, vibrotactile, and auditory stimulation. Horizontal lines indicate the performance level of $95 \%$.

all, performance levels of 3 of the 4 subjects (E1, E2, and N1) were either above or slightly below $95 \%$ correct at all rates tested with this relatively simple task.

A post hoc Scheffé $F$ test was conducted to examine intersubject differences. For motional stimulation, the Scheffé test indicated significant differences between Subjects E1 and N2 $(p<.05)$, Subjects E2 and N2 ( $p<$ $.05)$, and Subjects N1 and N2 $(p<.01)$; no significant differences were found between any pair of Subjects E1, E2, and N1. For vibrotactile stimulation, the Scheffé test again indicated significant differences between Subjects E1 and N2, Subjects E2 and N2, and Subjects N1 and N2 (all $p \mathbf{s}<.01$ ); significant differences were also found between Subjects E1 and E2 $(p<.01)$ and Subjects E1 and $\mathrm{N} 1(p<.05)$; and the difference between Subjects E2 and $\mathrm{N} I$ was not significant. For auditory stimulation, the
Scheffé test indicated a significant difference between Subjects N1 and N2 $(p<.05)$.

The Scheffé $F$ test was also used to examine performance differences due to stimulation modes. Significant differences $(p<.01)$ were found ( 1 ) between M (motional stimulation) and $\mathrm{V}$ (vibrotactile stimulation) for Subject E2, (2) between $M$ and V, $M$ and $A$ (auditory stimulation), and V and A for Subject N1, and (3) between $M$ and $A$, and $V$ and $A$ for Subject N2. These results suggested, at least for Subjects E2 and N1, a general increase in performance level as stimulation mode changed from motional to vibrotactile to auditory.

\section{Three-Letter Random-Sequence Identification}

The percent correct scores averaged over the last three runs for motional, vibrotactile, and auditory stimulation 
Table 2

Number of Runs Required to Reach Performance Criterion for the Two Training Tasks

\begin{tabular}{|c|c|c|c|c|c|c|}
\hline \multirow{2}{*}{$\begin{array}{l}\text { Presentation } \\
\text { Rate (wpm) }\end{array}$} & \multicolumn{3}{|c|}{ One-Letter (130 trials/run) } & \multicolumn{3}{|c|}{ Three-Letter ( 52 trials/run) } \\
\hline & M & $\mathrm{V}$ & $\mathrm{A}$ & M & $\mathrm{V}$ & A \\
\hline \multicolumn{7}{|c|}{ Subject E1 } \\
\hline 12 & 14 & 3 & - & 22 & 8 & - \\
\hline 16 & 5 & 4 & - & 27 & 5 & - \\
\hline 20 & - & 23 & - & 22 & 31 & - \\
\hline 24 & 33 & 16 & - & 27 & 30 & - \\
\hline \multicolumn{7}{|c|}{ Subject E2 } \\
\hline 12 & 12 & 1 & 一 & 12 & 3 & - \\
\hline 16 & 10 & 4 & - & 16 & 1 & - \\
\hline 20 & - & 5 & - & 38 & 4 & - \\
\hline 24 & 35 & 5 & - & 15 & 77 & - \\
\hline \multicolumn{7}{|c|}{ Subject N1 } \\
\hline 12 & 9 & 1 & 2 & 26 & 3 & 2 \\
\hline 16 & 7 & 3 & 1 & 32 & 6 & 2 \\
\hline 20 & - & 4 & 4 & 26 & 33 & 8 \\
\hline 24 & 34 & 16 & 1 & 7 & 19 & 7 \\
\hline \multicolumn{7}{|c|}{ Subject N2 } \\
\hline 12 & 19 & 4 & 1 & 109 & 15 & 4 \\
\hline 16 & 20 & 23 & 1 & 61 & 36 & 3 \\
\hline 20 & - & 27 & 1 & 6 & 19 & 6 \\
\hline 24 & 61 & 20 & 2 & 1 & 14 & 21 \\
\hline
\end{tabular}

Note-M, motional; V, vibrotactile; A, auditory.

are shown for individual subjects and presentation rates in Figure 5. The number of runs required to meet criterion performance for each subject, presentation rate, and stimulation mode is presented in Table 2 . With motional stimulation, performance levels of 3 subjects $(E 1, E 2$, and N1) were above $90 \%$ at rates up to $16 \mathrm{wpm}$. Their performance levels decreased as presentation rates increased beyond $16 \mathrm{wpm}$, with this effect being more pronounced for Subject N1. The performance levels of Subject N2 were lower at all rates tested. With vibrotactile stimulation, Subject E2 was the only subject whose performance levels were above $90 \%$ at all rates tested. The performance levels of Subjects E1 and N1 were quite similar: They were above $90 \%$ for rates up to $16 \mathrm{wpm}$ and decreased as rates increased above this rate. The performance level of Subject N2 was above $90 \%$ at $12 \mathrm{wpm}$ but dropped precipitously as rate increased. With auditory stimulation, both inexperienced subjects were able to reach the performance criterion at all rates tested and with much less training than was required for motional or vibrotactile stimulation, except for Subject N2 at 24 wpm (see Table 2; also see note 4). Thus, it is clear that (1) this training task was more difficult than the single-letter identification training for all subjects, (2) performance levels generally deteriorated as presentation rate increased, (3) performance of the inexperienced subjects with auditory stimulation was better than that with vibrotactile stimulation, and (4) performance with vibrotactile stimulation was better than that with motional stimulation. As mentioned previously, the experienced subjects were not tested with auditory stimulation because this task was trivial for them.
For motional stimulation, a post hoc Scheffé $F$ test on intersubject differences indicated significant differences between Subjects E1 and E2 $(p<.05)$ and between the other five subject pairs $(p s<.01)$. For vibrotactile stimulation, the Scheffé test indicated that among the six possible subject pairs, the difference between Subjects E1 and $\mathrm{N} 1$ was insignificant, whereas the differences between the other five pairs were significant $(p s<.01)$. For auditory stimulation, the Scheffé test indicated a significant difference between Subjects N1 and N2 $(p<.01)$.

A Scheffé $F$ test on performance differences due to stimulation modes indicated significant differences $(p<$ .01) (1) between $M$ (motional stimulation) and V (vibrotactile stimulation) for Subject E2, and (2) between M and $\mathrm{V}, \mathrm{M}$ and $\mathrm{A}$ (auditory stimulation), and $\mathrm{V}$ and $\mathrm{A}$ for each of Subjects N1 and N2. Except for Subject E1, the subjects' performance was significantly better with vibrotactile stimulation than with motional stimulation. In addition, the inexperienced subjects performed significantly better with auditory stimulation than with motional or vibrotactile stimulation.

\section{Common-Word Identification}

The percent correct scores averaged over the three runs tested per experimental condition for each subject, presentation rate, and stimulation mode are shown in the first three panels of Figure 6. In general, the word-identification performance of Subjects E1 and E2 was superior to that of Subjects N1 and N2 for each of the three types of stimulation. That is, at a given rate of presentation, the scores of Subjects E1 and E2 exceeded those of Subjects N1 and N2, and the psychometric functions of Subjects E1 and E2 extended over higher rates of presentation than did those of Subjects N1 and N2. Larger differences between the two subject groups were observed for auditory and vibrotactile conditions than for motional stimulation. (Had our apparatus been capable of testing motional stimulation at rates higher than $24 \mathrm{wpm}$, larger differences between the two subject groups might have been observed for this stimulation mode as well.)

Statistical analyses were applied to some of the data collected with motional and vibrotactile stimulation. For each of these two stimulation modes, the data consisted of those of the 4 subjects, the four stimulus presentation rates over which all subjects were tested (i.e., 12, 16, 20, and $24 \mathrm{wpm}$ ), and three repetitions per subject $\times$ rate combination. A post hoc Scheffé $F$ test on intersubject differences indicated significant differences $(p<.01)$ between all six possible subject pairs for each of the two stimulation modes. A Scheffé $F$ test on performance differences due to stimulation modes indicated significant differences $(p<.01)$ between motional and vibrotactile stimulation for each of the 4 subjects. In other words, all subjects performed significantly better with vibrotactile stimulation than with motional stimulation. From Figure 6 , it is obvious that all subjects performed better with auditory stimulation than with motional or vibrotactile stimulation. 
Motional Stimulation

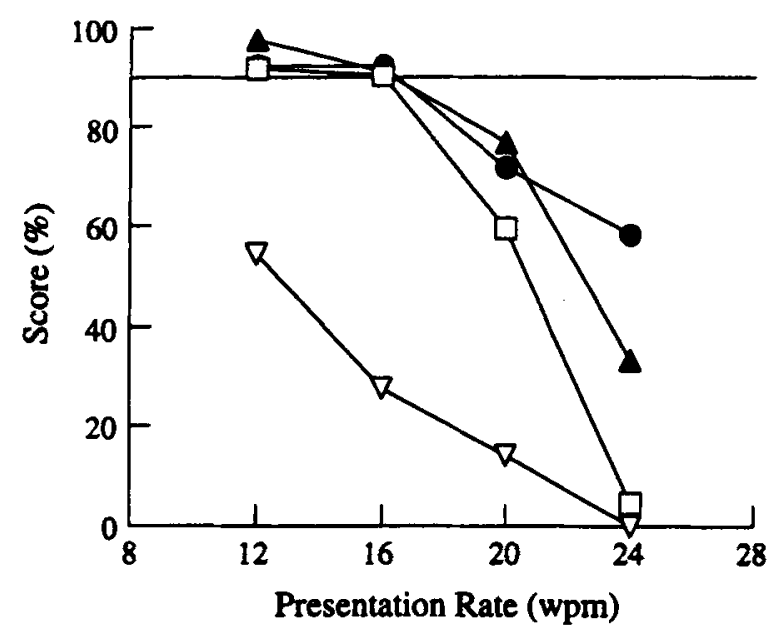

Auditory Stimulation

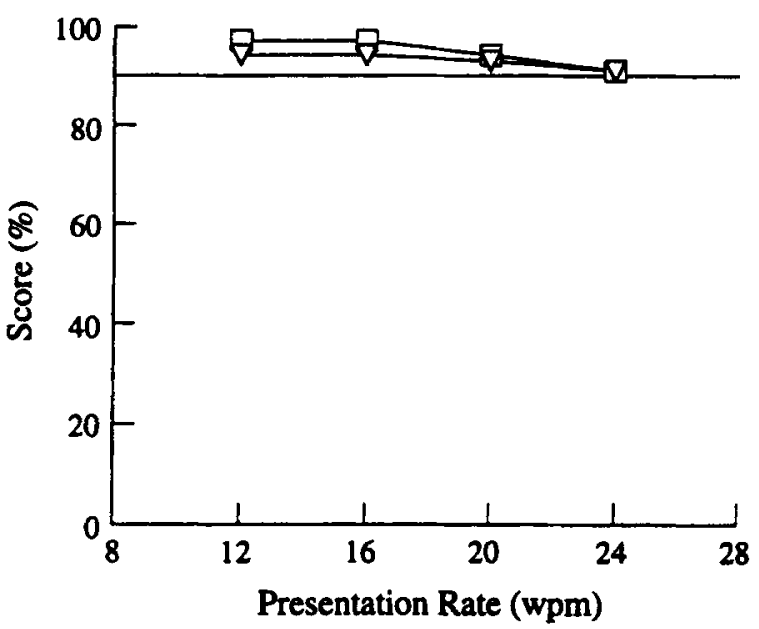

Vibrotactile Stimulation

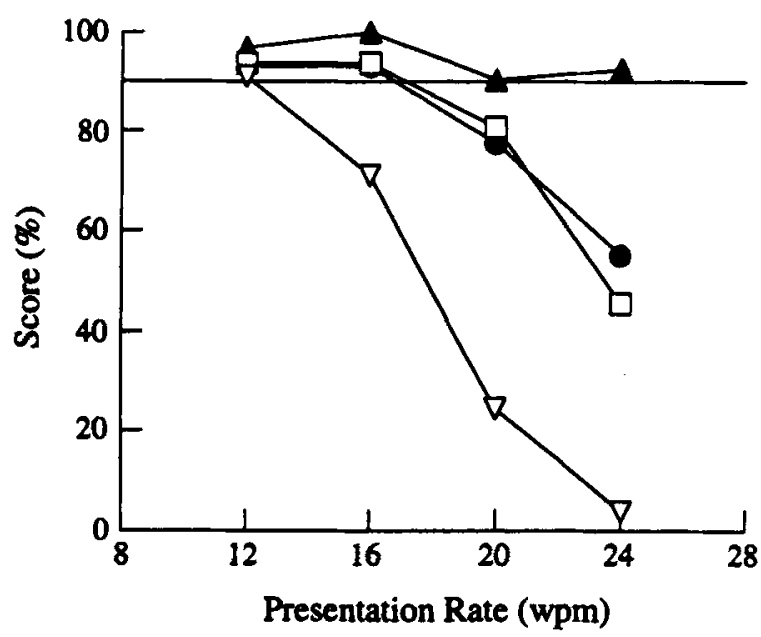

Figure 5. Percent correct scores averaged over the final three runs from three-letter random-sequence identification training as a function of presentation rate. Individual subject results are shown in separate panels for motional, vibrotactile, and auditory stimulation. Horizontal lines indicate the performance level of $90 \%$.

For motional and vibrotactile stimulation, a Scheffé test on the two subject groups indicated a significant difference $(p<.01)$ between the experienced and inexperienced subjects. It is also obvious from the lower left panel of Figure 6 that the experienced subjects performed better than the inexperienced subjects for auditory stimulation. Therefore, percent correct scores were averaged across the 2 experienced subjects (E1 and E2) and the 2 inexperienced subjects (N1 and N2) for motional (M), vibrotactile (V), and auditory (A) stimulation (final panel of Figure 6). It can be seen that performance decreased with stimulus presentation rate at an average rate of roughly $3 \%-5 \% / \mathrm{wpm}$ for the experienced subjects and $5 \%-7 \% /$ wpm for the inexperienced subjects. Extrapolated values of the presentation rates corresponding to a score of $50 \%$ correct were roughly $22 \mathrm{wpm}(\mathrm{M})$, $31 \mathrm{wpm}(\mathrm{V})$, and $51 \mathrm{wpm}(\mathrm{A})$ for the experienced subjects and $11 \mathrm{wpm}(\mathrm{M}), 16 \mathrm{wpm}(\mathrm{V})$, and $25 \mathrm{wpm}(\mathrm{A})$ for the inexperienced subjects.

As another metric summarizing performance, the equivalent word rate $\gamma$ was calculated as the product of percent correct score and stimulus presentation rate (Cholewiak, Sherrick, \& Collins, 1993, refer to this measure as the correct words per minute). A maximum $\gamma$ was associated with each stimulation mode and subject group (see Figure 7). As stimulus presentation rate increased, $\gamma$ increased initially, but it was limited by the highest achievable value (i.e., the presentation rate). Then, a region of presentation rates was observed where a tradeoff appeared to exist between presentation rate and percent correct scores: $\gamma$ remained near a maximum level with increasing presentation rate. Beyond this region, $\gamma$ decreased as presentation rate increased. The maximum $\gamma$ scores averaged across the experienced subjects were 14 , 
Motional Stimulation
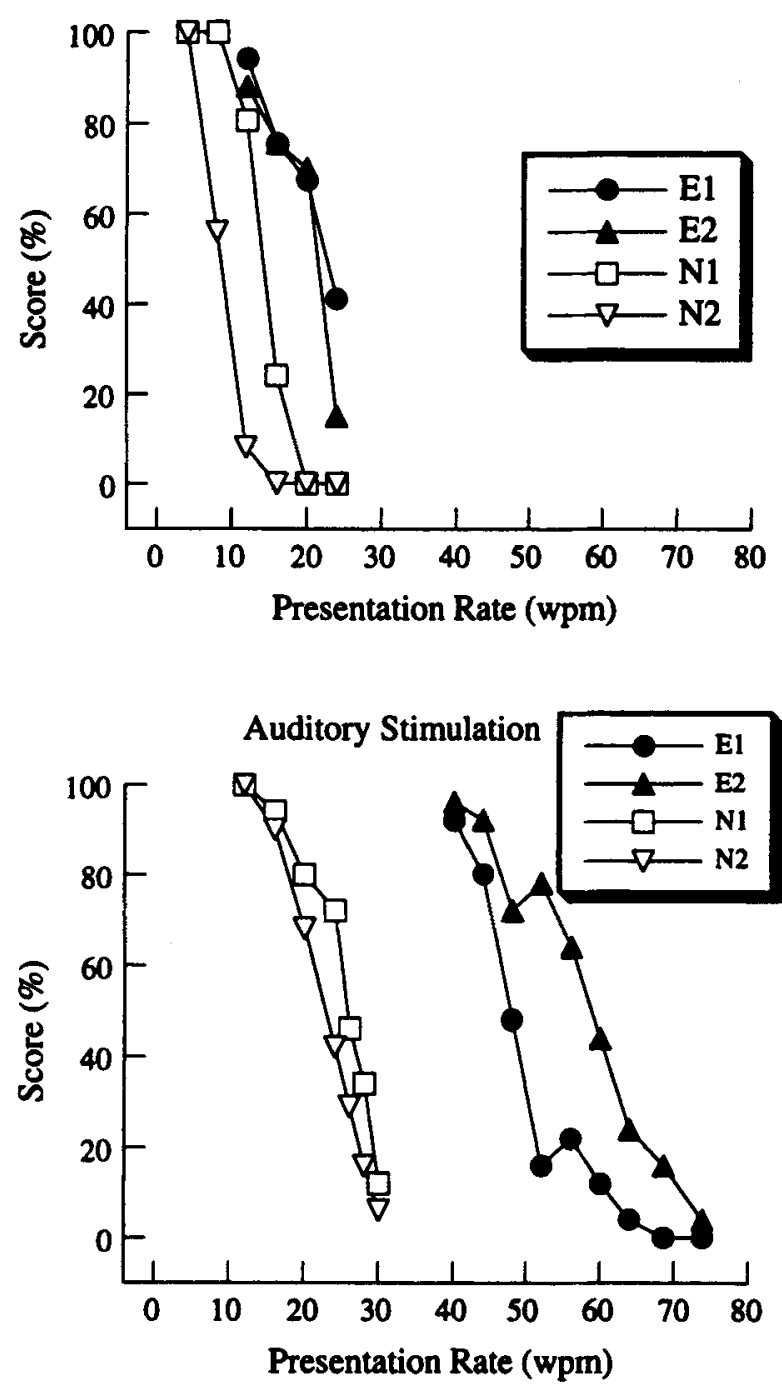
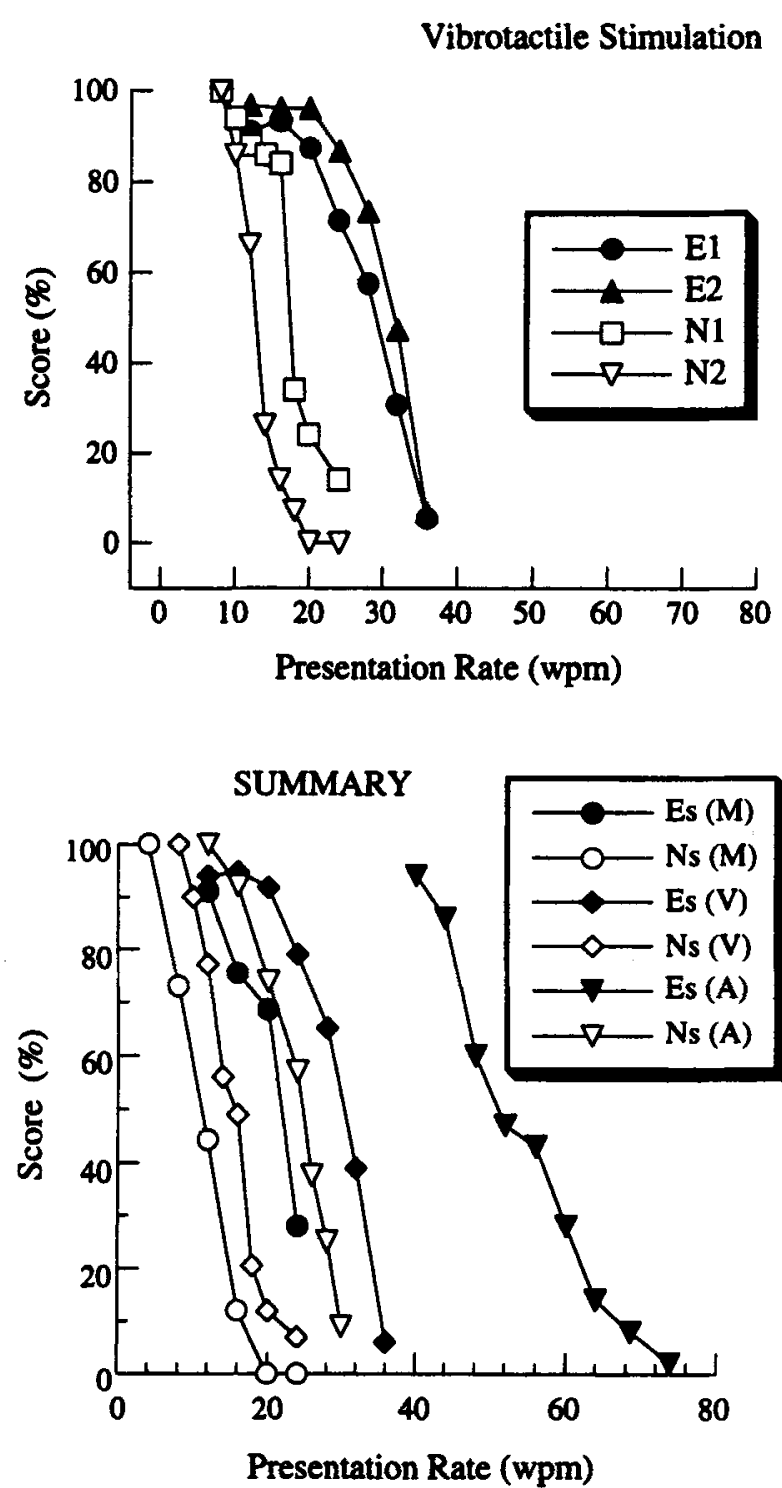

Figure 6. Percent correct scores averaged over the three runs from common-word identification test as a function of presentation rate. Individual subject results are shown in separate panels for motional, vibrotactile, and auditory stimulation. Also shown are averaged results in the summary panel.

19 , and 38 wpm with motional, vibrotactile, and auditory stimulation, respectively. The corresponding scores averaged across the inexperienced subjects were 6,9 , and $15 \mathrm{wpm}$, respectively.

\section{Sentence Reception}

The inexperienced subjects were unable to perform this test with any of the stimulation types; hence, only the experienced subjects were tested. The percent correct scores averaged over the three runs for motional (M), vibrotactile (V), and auditory (A) stimulation are shown for individual subjects and presentation rates in the first three panels of Figure 8. For all stimulation types, performance levels showed a general decreasing trend as stimulus presentation rate increased. Performance with auditory stimulation was substantially better than that with vibrotactile stimulation, which, in turn, was better than that with motional stimulation $(p<.01)$. From the summary graph (lower right panel of Figure 8 ), it was estimated that percent correct word scores decreased with stimulus presentation rate at average rates of about $6 \%$ / wpm for word scores near $50 \%$. Extrapolated values of the presentation rates corresponding to a score of $50 \%$ correct were $25 \mathrm{wpm}(\mathrm{M}), 32 \mathrm{wpm}(\mathrm{V})$, and $59 \mathrm{wpm}(\mathrm{A})$. The average maximum $\gamma$ scores were 18,21 , and $43 \mathrm{wpm}$ with motional, vibrotactile, and auditory stimulation, respectively. The slightly higher $\gamma$ achieved with this test compared with that achieved with the common-word identification test was probably due to the increased redundancy in the test material. 


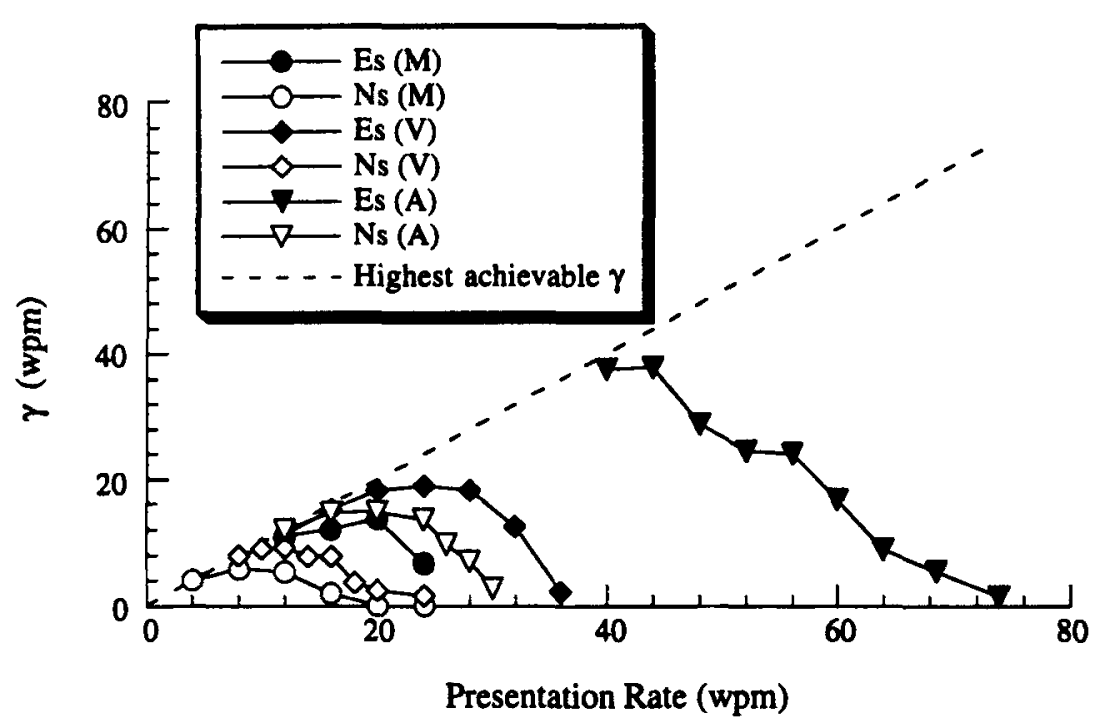

Figure 7. Equivalent word rates $\gamma(w p m)$ from common-word identification test for experienced (filled symbols) and inexperienced (open symbols) subjects with motional (circles), vibrotactile (diamonds), and auditory (triangles) stimulation. Dashed line indicates the highest achievable equivalent word rate (i.e., the presentation rate).

\section{DISCUSSION}

\section{Experienced Versus Naive Subjects}

For the training tasks of single-letter and three-letter random-sequence identification, Subjects E1, E2, and $\mathrm{N} 1$ performed at very similar levels in terms of the final percent correct scores achieved and the total number of runs needed to reach these scores. Subject N2 generally achieved lower scores at higher presentation rates and took longer to reach those scores. The performance differences between the two inexperienced subjects (N1 and N2) may be attributed to the additional experience of Subject N1 outside of the formal test sessions. As the experimenter, Subject NI was exposed to the test signals during experimental setup and debugging. Subject N2 was, therefore, the only subject who had no experience with Morse code or with motional stimulation prior to the experimental sessions. For these relatively simple tasks that required the subjects only to associate 26 motional, vibrotactile, or auditory patterns to the 26 letters of the English alphabet, prior knowledge of the Morse code did not necessarily result in better performance (i.e., $\mathrm{Nl} \approx$ Subjects $\mathrm{E} 1$ and $\mathrm{E} 2$ ). For the two testing tasks of word and sentence identification, however, the experienced subjects consistently performed better than did the inexperienced subjects as a group. Previous experience in receiving connected Morse code sequences through the auditory modality appears to have resulted in better performance on word and sentence identification tasks than that achieved by the inexperienced subjects in all three modalities. With that in mind and without loss of generality, we shall base the rest of this discussion on the results averaged over the experienced and inexperienced subjects, respectively.
In order to compare the subjects' performance across modalities and tasks, the equivalent word rates $(\gamma)$ were computed for all cases. These results are shown in Figure 9. The asterisks above the columns for the singleletter identification task indicate that these $\gamma$ values might have been higher if stimulus presentation rates over $24 \mathrm{wpm}$ had been used. On the average, excluding data from the single-letter identification task, the ratio of the equivalent word rates for vibrotactile stimulation to that for motional stimulation $\left(\gamma_{\mathrm{V}}: \gamma_{\mathrm{M}}\right)$ was 1.2 for the experienced subjects and 1.5 for the inexperienced subjects. The ratio of the equivalent word rates for auditory stimulation to that for motional stimulation $\left(\gamma_{\mathrm{A}}: \gamma_{\mathrm{M}}\right)$ was 2.6 for the experienced subjects and 2.5 for the inexperienced subjects. The ratio of the equivalent word rates for auditory stimulation to that for vibrotactile stimulation $\left(\gamma_{\mathrm{A}}: \gamma_{\mathrm{V}}\right)$ was 2.2 for the experienced subjects and 1.7 for the inexperienced subjects. Overall, for both subject groups, auditory reception rates were nearly twice as high as for vibrotactile stimulation, which in turn was about 1.3 times that for motional stimulation.

\section{Auditory Versus Tactual Reception}

The difference in the auditory and tactual rate of Morse code reception may be explained in terms of the unit signal length and the temporal properties of taction and audition. In general, the auditory system responds faster and more accurately to dynamic stimulation than does the tactual system. For instance, Gescheider (1966) reported that the time difference necessary for resolving two successive events was $1.8 \mathrm{msec}$ for equally loud binaural clicks and $10 \mathrm{msec}$ for pulses applied to the fingertip. Our results can be compared quantitatively with those obtained by Lechelt (1973) on auditory and tactile nu- 
Motional Stimulation

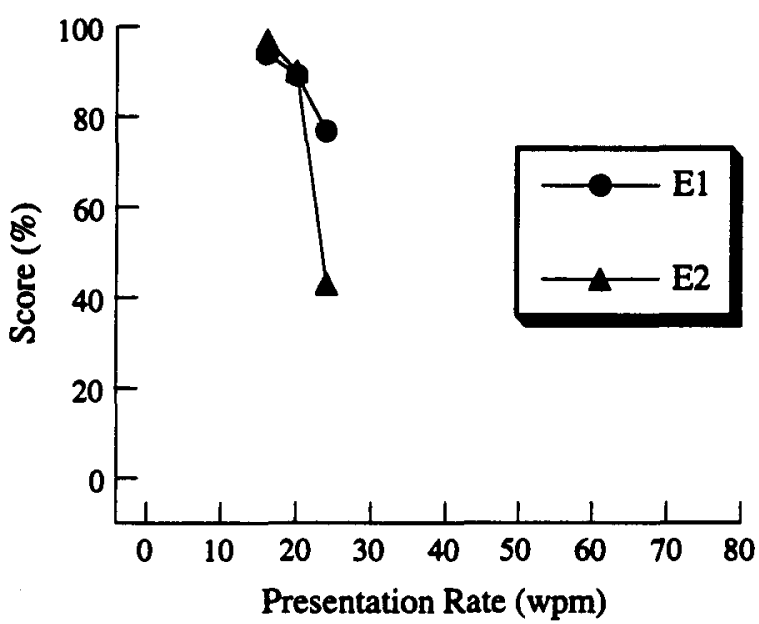

Auditory Stimulation

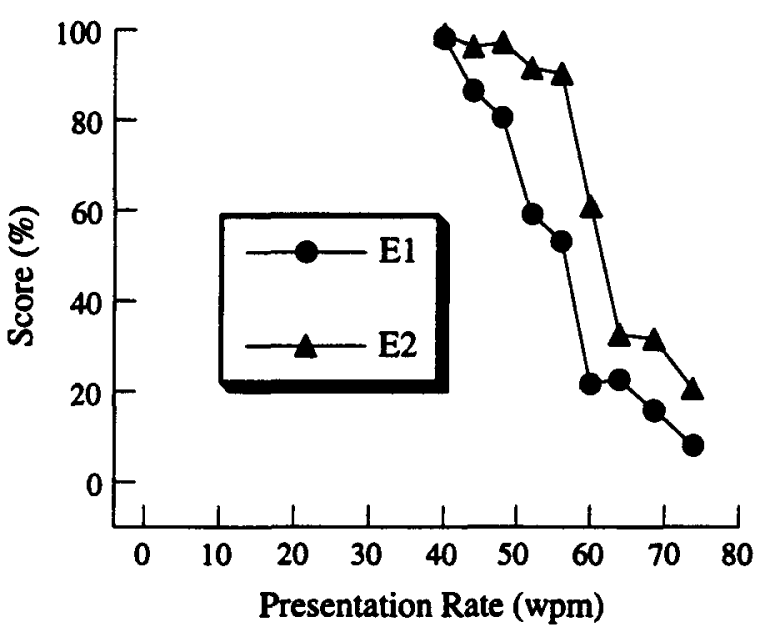

Vibrotactile Stimulation
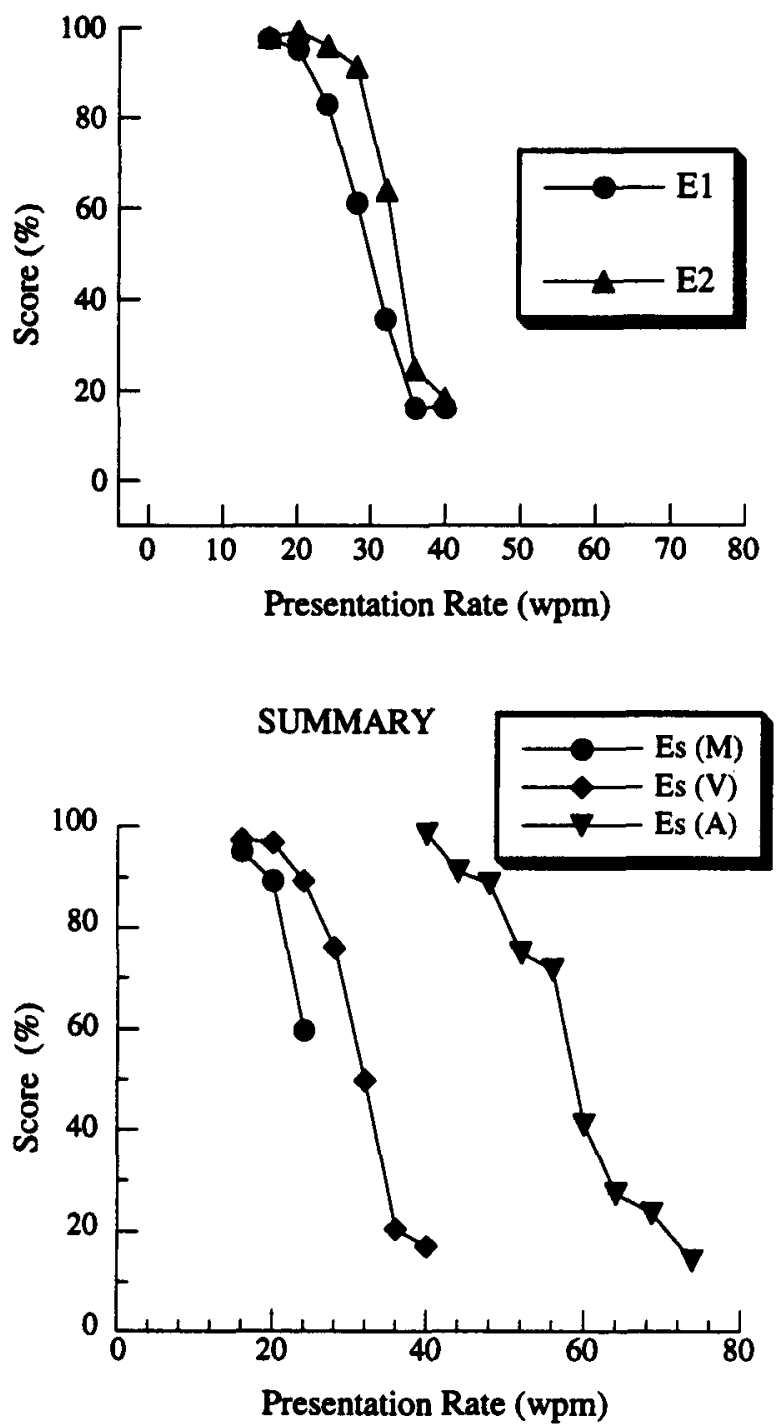

Figure 8. Percent correct scores averaged over the three runs from sentence reception test as a function of presentation rate. Data averaged across the experienced subjects are shown for motional (circles), vibrotactile (diamonds), and auditory (triangles) stimulation. Also shown are averaged results in the summary panel.

merousness perception using binaural clicks and 2-msec square-wave mechanical taps to the left middle finger with trains of 2-9 signals presented at rates of 3-8 items/ sec. Lechelt (1973) found that whereas auditory counts were nearly perfect for all conditions tested, cutaneous counts tended to underestimate the actual number of signals. Cutaneous counts were about $90 \%$ of the actual number of signals at a presentation rate of 8 items $/ \mathrm{sec}$. Simplifying the Morse code as a series of dots (e.g., the code for $\mathrm{H}$ is dit-dit-dit-dit), a constant rate of 8 items/ sec corresponds to $U=63 \mathrm{msec}$, or, equivalently, $19 \mathrm{wpm}$. Despite the difference in signal duty cycles between Lechelt's study and ours, this is consistent with the equivalent word rate of 18 and $21 \mathrm{wpm}$ with motional and vibrotactile stimulation, respectively, achieved by the experienced subjects.

\section{Equivalent-Word-Rate Comparisons}

The difference in performance between the two subject groups is evident in that whenever both subject groups performed the same tasks, the experienced subjects attained higher values of $\gamma$ than did the inexperienced subjects. The inexperienced subjects were simply unable to perform some of the tasks, despite the fact that each subject received a total of $70-80 \mathrm{~h}$ of training. The experienced subjects had more than 20 years of experience with Morse code, and this gave them several advantages over the inexperienced subjects. First, the experienced subjects were able to process stimuli at letter and word levels instead of individual signal components. The subjects reported that they could "hear" the code while feeling the motions or vibrations on their fingers. This transfer of learning from the tactual sense to the au- 

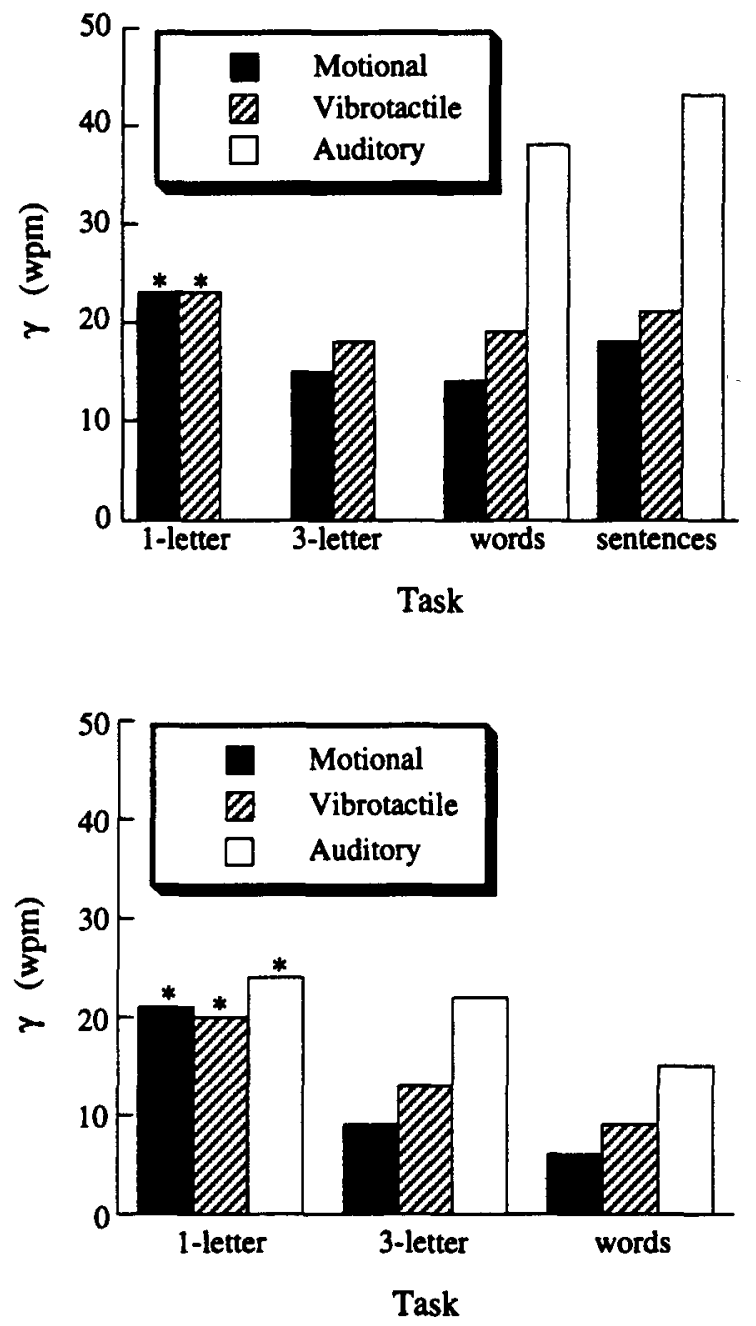

Figure 9. Equivalent word rates $\gamma(\mathrm{wpm})$ for each of the tasks and modes of stimulation. Upper panel presents results averaged across the 2 experienced subjects; lower panel presents results averaged across the 2 inexperienced subjects.

ditory sense, the modality on which these subjects were highly trained, allowed them to have more time to concentrate on the content of the message rather than focusing on the identification of single letters. Differences in the response strategy of the two subject groups for the common-word test material illustrate this point. The strategy of the inexperienced subjects was to type out the responses letter by letter and then edit the string of letters into meaningful words. The experienced subjects, however, would either type out a whole word or skip a trial if they failed at word recognition. These subjects occasionally made spelling errors indicating again that they were focusing on words rather than letters. Second, the experienced subjects were well trained with "chunking" of letters into meaningful words or messages. They reported that, during the reception of a word, they were constantly predicting the next letter on the basis of letters already presented. This ability to hold letters in short-term memory until they are incorporated into a meaningful unit is the result of years of Morse code practice. Finally, both of the experienced subjects used the straight key to send Morse code element by element before the more efficient iambic keyer became available. Their ability to send Morse code manually might have contributed to their ability to receive the code tactually.

To follow up the last point, a supplementary test was performed to determine the speed at which the experienced subjects could send Morse code. They were tested with the straight key since its element-by-element mode corresponds directly to the mode used in our reception tests. The resulting speed for manually sending the Morse code of CUNY sentences was $23 \mathrm{wpm}$ for each experienced subject. ${ }^{5}$ This is consistent with the equivalent word rates obtained from sentence-reception tests with motional and vibrotactile stimulation (18 and $21 \mathrm{wpm}$, respectively; see the top panel of Figure 9) for these experienced subjects.

\section{Comparison With Other Methods of Tactual Communication}

The information transfer rates of several tactual communication methods can be compared. For natural methods of tactual communication, Reed, Durlach, and Delhorne (1992) estimated information transfer rates to range from $7.5 \mathrm{bits} / \mathrm{sec}$ for fingerspelling to $12-14 \mathrm{bits} / \mathrm{sec}$ for Tadoma and tactual sign language. On the basis of the present results, the information transfer rate for receiving Morse code using conversational English material through motional and vibrotactile stimulation is roughly 2.7 bits/sec. ${ }^{6}$ Foulke and Brodbeck (1968) reported that experienced Morse code operators were able to receive the code by electrocutaneous stimulation at a rate of $10 \mathrm{wpm}$, or roughly $1.3 \mathrm{bits} / \mathrm{sec}$ (according to note 6 ). These relatively low rates of tactual reception of Morse code are most likely limited not only by subjects' reception rate but by major inefficiencies in the code--that is, the bitwise coding of information, the 3:1 dash-dot ratio, and the relatively long silences between dots and dashes. With the standard timing pattern for Morse code, the average duration across the 26 letters is roughly $8 U$. At a presentation rate of $20 \mathrm{wpm}(U=60 \mathrm{msec})$, the average duration for a letter is $480 \mathrm{msec}$.

Using his pneumatic reverse typewriter, Bliss (1961) reported that one experienced typist was able to receive 26 letters and several punctuation symbols at a rate of $4.5 \mathrm{bits} / \mathrm{sec}$, with a stimulus presentation rate of $1.32 \mathrm{sym}$ bols $/ \mathrm{sec}$ and a stimulus uncertainty of $4.9 \mathrm{bits} /$ presentation. Using the Optacon device (Linvill \& Bliss, 1966) and English sentences as test material, Cholewiak et al. (1993) reported that their best subject was able to reach a word rate of $40 \mathrm{wpm}$, or $5.4 \mathrm{bits} / \mathrm{sec}$. Using the display for the Vibratese language, Geldard (1957) reported that 1 subject was able to handle $38 \mathrm{wpm}$, or $5.1 \mathrm{bits} / \mathrm{sec}$. These information-transfer rates are higher than those obtained here for Morse code. In making such a comparison, however, it should be noted that whereas our apparatus conveyed Morse code through a 1-bit display, Bliss's device employed eight finger rests with an average stimulus un- 
certainty of 2.2 bits per finger, the Optacon consisted of $108(6 \times 18$, according to Figure 47-1 in Cholewiak et al., 1993) 1-bit stimulating pins, and Vibratese was coded using five vibrators with 3 bits per vibrator.

We are currently investigating the feasibility of communicating through combined tactile and kinesthetic stimulation on multiple fingers using a novel multifinger positional display. It is hoped that by improving the encoding scheme as well as the display, we can achieve information rates comparable to those demonstrated by natural methods of tactual communication.

\section{REFERENCES}

The ARRL Handbook for Radio Amateurs (7th ed.). (1993). Newington, CT: American Radio Relay League.

BuIss, J. C. (1961). Communication via the kinesthetic and tactile senses. Unpublished doctoral dissertation, MIT, Department of Electrical Engineering.

Bryan, W. L., \& Harter, N. (1899). Studies on the telegraphic language: The acquisition of a hierarchy of habits. Psychological Review, 6, 345-375.

Boothroyd, A., Hanin, L., \& Hnath, T. (1985). A sentence test of speech perception: Reliability, set equivalence, and short term learning (Speech and Hearing Science Report No. RC110). New York: City University of New York.

Carroll, J. B., Davies, P., \& Richman, B. (1971). The American Heritage word frequency book. New York: American Heritage.

Cholewiak, R. W., Sherrick, C. E., \& Collins, A. A. (1993). Princeton cutaneous research project (Report No. 62). Princeton, NJ: Princeton University.

Clark, F. J., \& HorCh, K. W. (1986). Kinesthesia. In K. R. Boff, L. Kaufman, \& J. P. Thomas (Eds.), Handbook of perception and human performance: Sensory processes and perception (Vol. 1, pp. 13/1-13/62). New York: Wiley.

Eberhardt, S. P., Coulter, D. C., Bernstein, L. E., Barac-Cikoja, D., \& JORDAN, J. (1994). Inducing dynamic haptic perception by the hand: System description and some results. In C. J. Radcliffe (Ed.), Proceedings of the Winter Annual Meeting of the American Society of Mechanical Engineers: Dynamic Systems and Control (Vol. 55, pp. 345-351). New York: American Society of Mechanical Engineers.

Foulke, E., \& BRodBecK, A. A., JR. (1968). Transmission of Morse code by electrocutaneous stimulation. Psychological Record, 18, 617-622.

Geldard, F. A. (1957). Adventures in tactile literacy. American Psychologist, 12, 115-124.

GELDARD, F. A. (ED.) (1973). Cutaneous communication systems and devices. Austin, TX: Psychonomic Society.

GESCHEIDER, G. A. (1966). Resolving of successive clicks by the ears and skin. Journal of Experimental Psychology, 71, 378-381.

Gescheider, G. A., Hoffman, K. E., Harrison, M. A., Travis, M. L., \& BolANOWSKI, S. J. (1994). The effects of masking on vibrotactile temporal summation in the detection of sinusoidal and noise signals. Journal of the Acoustical Society of America, 95, 1006-1016.

KaCZmareK, K. A., Webster, J. G., BaCh-Y-Rita, P., \& Tompkins, W. J. (1991). Electrotactile and vibrotactile displays for sensory substitution systems. IEEE Transactions on Biomedical Engineering, 38, 1-15.

LECHELT, E. C. (1973). Some stimulus parameters of tactile numerousness perception. In F. A. Geldard (Ed.), Cutaneous communication systems and devices (pp. 1-5). Austin, TX: Psychonomic Society.

LINVILL, J. G., \& Bliss, J. C. (1966). A direct translation reading aid for the blind. Proceedings of the Institute of Electrical \& Electronics Engineers, 54, 40-51.

ReEd, C. M., Durlach, N. I., \& Delhorne, L. A. (1992). The tactual reception of speech, fingerspelling, and sign language by the deafblind. Digest of Technical Papers of the Society for Information Display International Symposium, 23, 102-105.

Shannon, C. E. (1951). Prediction and entropy of printed English. Bell System Technical Journal, 30, 50-64.
VERRILlo, R. T., \& SMith, R. L. (1976). Effect of stimulus duration on vibrotactile sensation magnitude. Bulletin of the Psychonomic Society, 8, 112-114.

\section{NOTES}

1. We use the letters $E$ (experienced) and $N$ (naive) to refer to subject's prior knowledge of Morse code. One more experienced and 2 more inexperienced subjects began the experiments but dropped out before sufficient data were collected.

2. Because each word could be presented twice, the subjects were not shown the correct word when a mistake was made.

3. The subjects were discouraged, but not prohibited, from experimenting with nonstandard settings. They were asked to document all deviations from the standard setup in a log book and to discuss them with the experimenter at the end of the session. For the single-letter identification experiment, Subject El used downward motions at the rates of 12 and $16 \mathrm{wpm}$ but switched to upward motions after starting the 24-wpm condition. For all subsequent experiments, a set of waveforms with a polarity opposite to that shown in Figure 2 was used for Subject E1. In addition, Subject E1 switched to a smaller range of motion (i.e., fingertip displacement was decreased to $5 \mathrm{~mm}$ ) to reduce fatigue in the three-letter random-sequence identification experiment. Subject E2 used the standard posture but preferred a larger range of motion after beginning the $24-\mathrm{wpm}$ condition. For all subsequent experiments, fingertip displacement was increased to $15 \mathrm{~mm}$ for Subject E2. It is not clear whether or not these subjects would have eventually reached the same performance level if they had been required to use only the standard postures and parameter settings. Since this was the first time that motional reception of Morse code was attempted, we felt that the subjects should be allowed to choose whatever method they felt comfortable with, as long as they used the method consistently.

4. Had the experienced subjects performed this task with auditory stimulation, they would have achieved nearly perfect scores at all rates tested.

5. In these tests, the subjects used a straight key oscillator (MFJ-557 from Tucker Electronics \& Computers), the output of which was connected to a cassette recorder. Each subject was asked to send manually the Morse code of five CUNY sentence lists. They were instructed to (1) send as fast as they could assuming an excellent receiver, (2) not correct for any mistakes, and (3) take breaks only between sentences. The recording was then timed and scored by another ham radio operator. The sending speed for each sentence was computed as the number of words sent divided by total time. The results for individual sentences were then averaged and multiplied by the overall percent correct scores.

6 . The information transfer rate was estimated as follows. The CUNY sentences contain 102 words per 12 sentences, thus averaging 8.5 words/ sentence. According to Shannon (1951, Figure 4), strings of that length have between 1.2 and 2.1 bits/letter. Using 2 bits/letter as the upper bound and 4 letters/word (from CUNY sentence statistics) as the average word length in the corpus, we estimated the information content to be 2 bits/letter $\times 4$ letters/word, or 8 bits/word. Assuming that the experienced subjects can receive Morse codes of CUNY sentences reliably at $20 \mathrm{wpm}$ (see top panel of Figure 9) through motional and vibrotactile stimulation, we conclude that the information transfer rate is $8 \mathrm{bits} /$ word $\times 20 \mathrm{wpm}$, or, equivalently, $2.7 \mathrm{bits} / \mathrm{sec}$.

\section{APPENDIX}

\section{The International Morse Code}

The International Morse Code is the original modulation method used in amateur radio. The two basic elements of Morse code are dot (sounded dit) and dash (sounded dah). It is usually received auditorily with fixed-frequency tones (usually between 500 and $1500 \mathrm{~Hz}$ ) indicating the presence and timing of dits and dahs. Unique combinations of dits and dahs specify the letters of the alphabet, numerals, punctuation marks, and procedure signals. For this study, we used letters only. A complete list of Morse code for letters appears in Figure A1 with short and long bars indicating dits and dahs, respectively. 


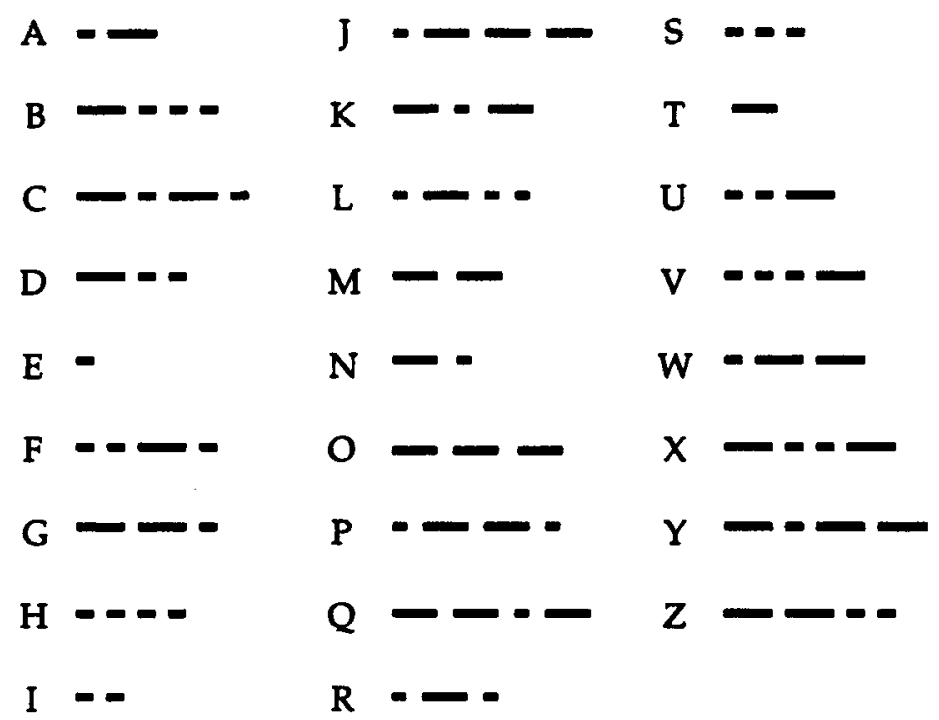

Figure A1. Morse code for letters of the English alphabet.

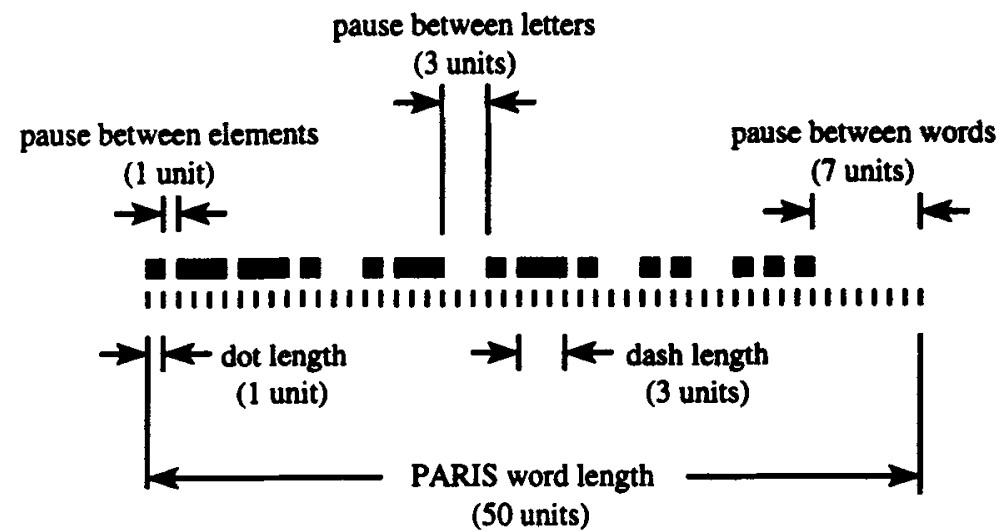

Figure A2. Diagram of timing in International Morse Code. From The $A R R L$ Handbook for Radio Amateurs (7th ed.), 1993, Newington, CT: American Radio Relay League. Copyright 1993 by the American Radio Relay League. Adapted with permission.

The length of a dit, $U$, is the basic unit of time in Morse code. The duration of a $d a h$ is $3 U$. Within a letter, the pause between adjacent elements is $U$. The space between letters is $3 U$. The space between words or groups is $7 U$. These relationships are illustrated in Figure A2.

The rate of Morse code is expressed in terms of words per minute (wpm). The length of a "standard" word is defined as $50 U$. The word Paris is of this length and is used to accurately set transmission speed. The relationship between the length of a dit, $U$, and the rate of transmission, $R$, is

$$
U(\mathrm{sec})=60 /[R(\mathrm{wpm}) \times 50],
$$

or, equivalently,

$$
U(\mathrm{msec})=1,200 / R(\mathrm{wpm}) .
$$

For instance, at $12 \mathrm{wpm}$, the duration of a dit is $100 \mathrm{msec}$ and that of a dah is $300 \mathrm{msec}$.

(Manuscript received November 6, 1995 revision accepted for publication November 11, 1996.) 